\title{
Comparison of Fluorescence Correlation Spectrometry with the Ordinary Fluorescence Optical Configuration with Flow Cytometry as a Tool for Micrometer-level Size Determination
}

\author{
Kitao FujIwara, ${ }^{\dagger}$ Shun Hirokawa, and Motohide AOKI \\ School of Life Sciences, Tokyo University of Pharmacy and Life Sciences, \\ 1432-1 Horinouchi, Hachiouji, Tokyo 192-0392, Japan
}

\begin{abstract}
As a tool for micrometer-level size determination, fluorescence correlation spectroscopy (FCS), performed based on our previous report, was compared to flow cytometry (FCM). For this purpose, standard fluorescent beads were subjected to both methods. And hence, it was found that our FCS is a useful method with satisfactory precision for size determinations of individual particles at micrometer size levels, while providing the average size for a mixture of two kinds of particles with different sizes.
\end{abstract}

(Received September 1, 2008; Accepted October 10, 2008; Published December 10, 2008)

In the previous paper, simple (inexpensive) FCS was proposed In this method, standard optical configuration is sufficient to observe whole cells, such as algae, whereas the confocal optical configuration was adopted for size determination at the molecular level. ${ }^{1}$ The movement of objects was effectuated using a stirrer, and was not attributable to Brownian motion; this method was also used to investigate the signal responses to different pinhole sizes. In this study, we performed size calibration of our system, and compared its efficacy to that of FCM with regard to algal size determination. Further, it is important to mention the limitation of our system in which the movement of an object is attributable to the stirrer in the case of algal cells

Many studies that applied FCS have recently been presented to the field of biochemistry. ${ }^{2-9}$ Further, a new technical approach has been attempted in FCS for increasing the amount of information obtained, such as the cross-correlation or fluorescence resonance energy transfer (FRET) techniques, ${ }^{10-12}$ including 2-photon processes. ${ }^{13,14}$ Successful imaging of the FCS signal is a recent challenge in this field of study. ${ }^{15,16}$

In most studies related to FCS, a confocal optical configuration has been adopted, because the observation volume should be less than a few fL for observing molecules. On the other hand, we have previously shown that a regular optical configuration in fluorescence spectrometry is adequate for the size determination of particles of size larger than $0.1 \mu \mathrm{m} .{ }^{1}$

For evaluating the result obtained by our system, we adopted FCM. Presently, FCM has become a powerful optical analytical tool for natural water planktons; this tool was first introduced by Trask et al. ${ }^{17}$ The FCM system was developed as an optical plankton analyzer. ${ }^{18,19}$ In the case of using FCM, the damage of DNA was inspected by irradiation of the light source of a laser. ${ }^{20}$ FCM was also employed to observe respiratory activities of marine bacterioplanktons. ${ }^{21}$ Following the commercialization of a portable field FCM specialized for the exploration and monitoring of particulates existing in natural water

† To whom correspondence should be addressed.
(FLOWCAM ${ }^{\circledR}$, Fluid Imaging Technology), diverse information on the ecology in natural water samples is now available. Therefore, it is important to compare the results on the efficacy of algal size determination by FCS following the method proposed in this study and FCM.

\section{Experimental}

\section{Reagents}

Fluoresbrite microspheres (Polyscience Inc. POL-YG series) were used as fluorescent particle standards. The surface of these latex particles was modified by fluorophores; the particles were stably suspended in an aqueous medium. We confirmed that the standard demonstrated maximum emission at $650 \mathrm{~nm}$ for an excitation wavelength of $325 \mathrm{~nm}$. In FCM, the size calibration standard was purchased from Molecular Probes (\#F-13838; Oregon, USA), and was additionally used to evaluate the size of fluorescent beads (Fluoresbrite microspheres). In the FCS measurements, the concentration of the particles was approximately $1 \mu \mathrm{g} / \mathrm{mL}$ for all of the bead samples; a weightbase suspension was used for the sake of adjusting the level of the fluorescence intensity. Based on the assumption that the specific gravity of latex (bead matrix) is almost equal to that of water, the concentrations of beads were $2.4 \times 10^{5}$ particles $/ \mathrm{mL}$ for $2-\mu \mathrm{m}, 8.8 \times 10^{3}$ particles $/ \mathrm{mL}$ for $6-\mu \mathrm{m}$, and $5.8 \times 10^{2}$ particles/mL for $10-\mu \mathrm{m}$, respectively.

\section{FCS measurement system}

The optical measurement system used was the same as that used in a previous paper on standard fluorometry. The emission from the cell was directly detected by a photon counter (Hamamatsu, Model C8855) through a pinhole (diameter $=50$ $\mu \mathrm{m})$ with $350-\mathrm{nm}$ high-pass and $650-\mathrm{nm}$ band-pass filters (Edmund Optics). The diameter of the laser beam was approximately $0.7 \mathrm{~mm}$. Therefore, the observation volume was calculated to be approximately $1.5 \times 10^{-9} \mathrm{~L}$. The signal was processed by a real-time correlator (ALV-multiple tau digital 
real correlator 5000/60XO; ALV-GmbH, Germany), in which the diffusion time was calculated based on the software of the real-time correlator with the attached software for 3-D diffusion, providing values of the diffusion time. A FCS measurement was performed for $180 \mathrm{~s}$ (observation time of $3 \mathrm{~s}$ with 60 accumulation) for each sample, and the diffusion time for each measurement was calculated using the curve-fitting function of the real-time correlator (in this case, data points from 20 to 215 were used for curve fitting). The diffusion time was measured 5 times, and the average and standard deviations of the diffusion time were obtained.

\section{Flow cytometry}

The size of the fluorescent particle standards and microalgae were determined from the forward scatter signals (wavelength, $480 \mathrm{~nm}$ ) obtained using FCM (FACSort; Becton Dickinson, NJ).

\section{Cultivation of microalgae}

The same unicellular freshwater algae as examined in a previous paper, Synechocystis sp. PCC 6803 and Chlorella kessleri C-531, were used as the planktonic observation targets; the algae were obtained from the IAM Culture Collection at the Institute of Molecular and Cellular Biosciences, University of Tokyo. Synechocystis cells were cultivated in a BG-11 medium (Allen (1968)) buffered with $0.03 \mathrm{~mol} \mathrm{~L}^{-1}$ HEPES-NaOH $(\mathrm{pH}$ 7.5) and suspended in $0.005 \mathrm{~mol} \mathrm{~L}^{-1} \mathrm{D}(+)$-glucose at $34^{\circ} \mathrm{C}$ under

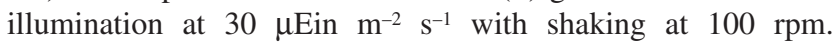
C. kessleri cells were cultivated in Gamborg's B5 medium. Gamborg's B5 medium (commercial solution purchased from Wako Pure Chemical Industries Ltd.) was diluted 5-fold with a $0.02 \mathrm{~mol} \mathrm{~L}^{-1}$ HEPES buffer (Wako) and $0.005 \mathrm{~mol} \mathrm{~L}^{-1} \mathrm{NaOH}$. Further, $\mathrm{D}(+)$-glucose was added, and the mixture was autoclaved. Incubation was performed at $25^{\circ} \mathrm{C}$ under

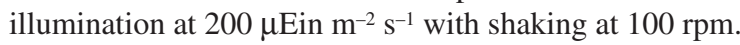

Both algae were cultivated for $48 \mathrm{~h}$ after seeding (cells were in the logarithmic phase; optical density (o.d.) $=0.5$ and 0.7 at 730 $\mathrm{nm}$ for Synechocystis sp. and C. kessleri, respectively) and were observed using the system described in this study.

\section{Results and Discussion}

Figure 1 shows calibration curve based on diffusion times of 2-, $6-$, and $10-\mu \mathrm{m}$ sized fluorescent particles in the present FCS Using this calibration curve, the sizes (diameter) of the Synechocystis sp. and C. kessleri cells were calculated as $2.7 \pm$ 0.1 and $7.3 \pm 0.2 \mu \mathrm{m}$, respectively. The exact sizes of both algae could be determined, although an approximate size estimation was based solely on the height of the $G(\tau)$ : FCS function value provided in our previous paper. ${ }^{1}$ Figures 2 - 5 show the FCM data. Using these data, the mean and standard deviation values of size, as shown in Figs. 4 and 5, were calculated to be $2.8 \pm$ 1.3 and $8.4 \pm 4.5 \mu \mathrm{m}$ for the Synechocystis sp. and C. kessleri cells, respectively. In the FCM, the size values of the Fluoresbrite standards $(2,6$, and $10 \mu \mathrm{m})$, which were used for the FCS measurements, were observed to be $1.9 \pm 0.1,6.1 \pm 0.3$, and $9.5 \pm 0.4 \mu \mathrm{m}$, respectively. Also, Figs. 2 and 3 show that the distribution in the size of Fluoresbrite (fluorescent standard) beads is not a normal one, at which the center is the assigned size. According to the precision indicated in Figs. 1 and 2, it can be concluded that the results obtained in the present FCS were as satisfactory as that obtained by FCM. Moreover, a Fluoresbrite particle (spherical polystyrene) was valid as a size standard for both algae.

In the data obtained with FCM, the size distribution of

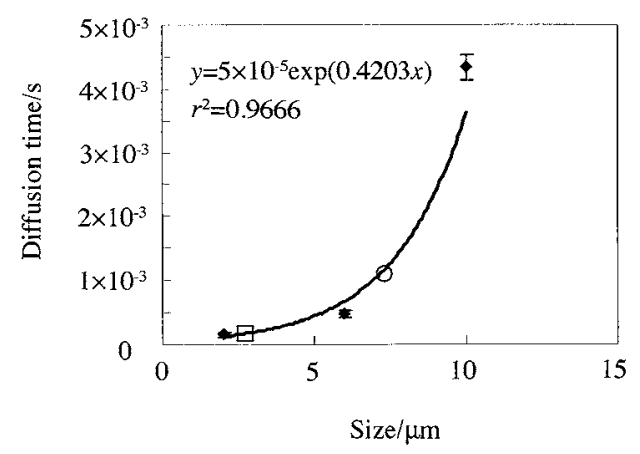

Fig. 1 Calibration curves for size determination. Diamond dot, standard beads; open circle, Chlorella kessleri; open square, Synechocystis sp. The function in the figure was employed for a fitting curve of the size calibration. Diffusion time (average $\pm \sigma$ ), ms: standard $(2 \mu \mathrm{m}), 0.15 \pm 0.039 ;$ standard $(6 \mu \mathrm{m}), 0.47 \pm 0.057$ standard $(10 \mu \mathrm{m}), 4.5 \pm 0.20 ;$ Synechocystis sp., $0.16 \pm 0.037$; Chlorella kessleri, $1.1 \pm 0.20$. $\sigma$, Standard deviation.

C. kessleri cells was considerably higher than that of Synechocystis sp. cells in solution. However, this deviation is not reflected in the FCS data. Similar results were obtained during size determinations of fluorescent beads, i.e., the deviation of the size determined by the present FCS was not as much as those by the FCM.

Figure 6 presents the FCS function $(G(\tau))$ for several bead standards (Fluoresbrite) and a mixture of 2 - and $10-\mu \mathrm{m}$ bead standards. The mixing ratio based on the number of particles for 2- and $10-\mu \mathrm{m}$ beads was about 1:1 (weight-base concentration) or 75:1 (particle-number-base concentration). As observed from the figure, the FCS signal of this mixture is that acquired using the $5-\mu \mathrm{m}$ size standard $(5.2 \pm 0.3 \mu \mathrm{m}$ in the calibration of the diffusion time). In the system investigated here, the observation period for calculating the diffusion time is extremely long, or the speed of movement of the objective particles is extremely high. Therefore, it is obvious that the average diffusion time of particles in the mixture was obtained by using the present system. In the FCM, the detection part of the optical signal is made of concentric circles of two laminar flows, where the center flow is a sample suspension of which the size was controlled by the outer flow. Although a study reported that a mixture of different-sized molecules (particles) yields a FCS curve (2-step curve) that is different from that yielded by the respective original molecules (particles), ${ }^{22}$ our system yielded the same FCS curve as that when a simple intermediate-size particle was employed. In cases of size determination in FCM, the magnitude of front scattering was used, while the FCS observed the movement speed of particles within the observation volume. For overcoming the defect of our system, single flow of the objective cell was indispensable.

In the present FCS system, pico- $(<3 \mu \mathrm{m})$, nano- $(3-20 \mu \mathrm{m})$, and micro- $(>20 \mu \mathrm{m})$ phytoplanktons, ${ }^{23,24}$ the existence of which is one of the current topics of research in environmental oceanography, are suitable targets for the present FCS optical configuration, since these phytoplanktons have a volume ranging from 15 to $4000 \mathrm{fL}$. However, at present, our system cannot be applied to actual samples containing algae of various sizes. The use of hydro flow can be one of the resolutions, as shown in initial FCS studies (micro-flow systems). ${ }^{25,26}$

As mentioned above, the observation volume in FCS under a confocal optical configuration conditions is extremely small, i.e., $1 \mathrm{fL}$ (represented as $\mathrm{V}_{\mathrm{CNE}}$ in Kunst et al.). ${ }^{26}$ This volume is 

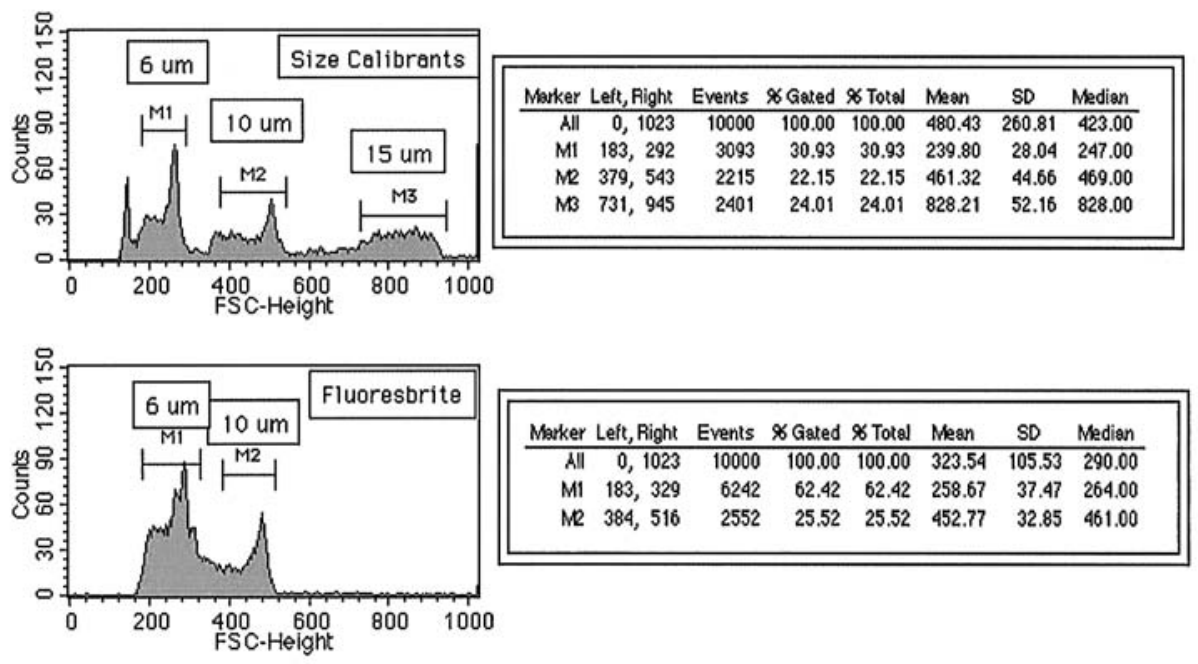

Fig. 2 Flow cytometry of fluorescent beads (Fluoresbrite). Data on the upper figure, size standard. Data on the lower figure, Fluoresbrite.
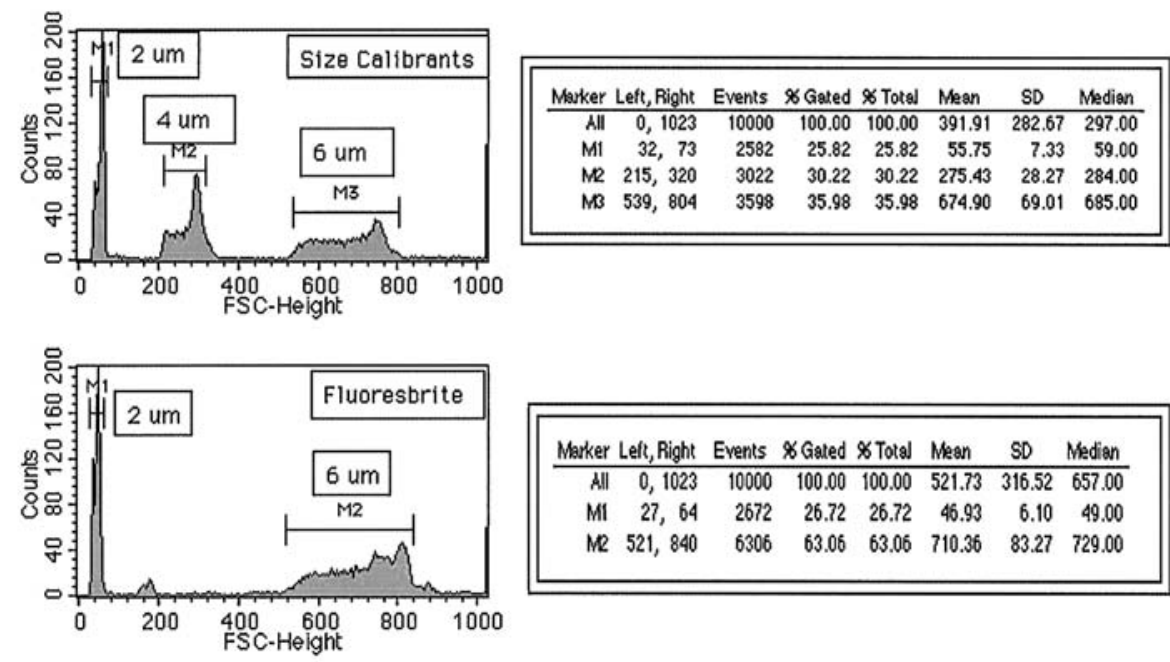

Fig. 3 Flow cytometry for fluorescent beads (Fluoresbrite). Data on the upper figure, size standard. Data on the lower figure, Fluoresbrite.
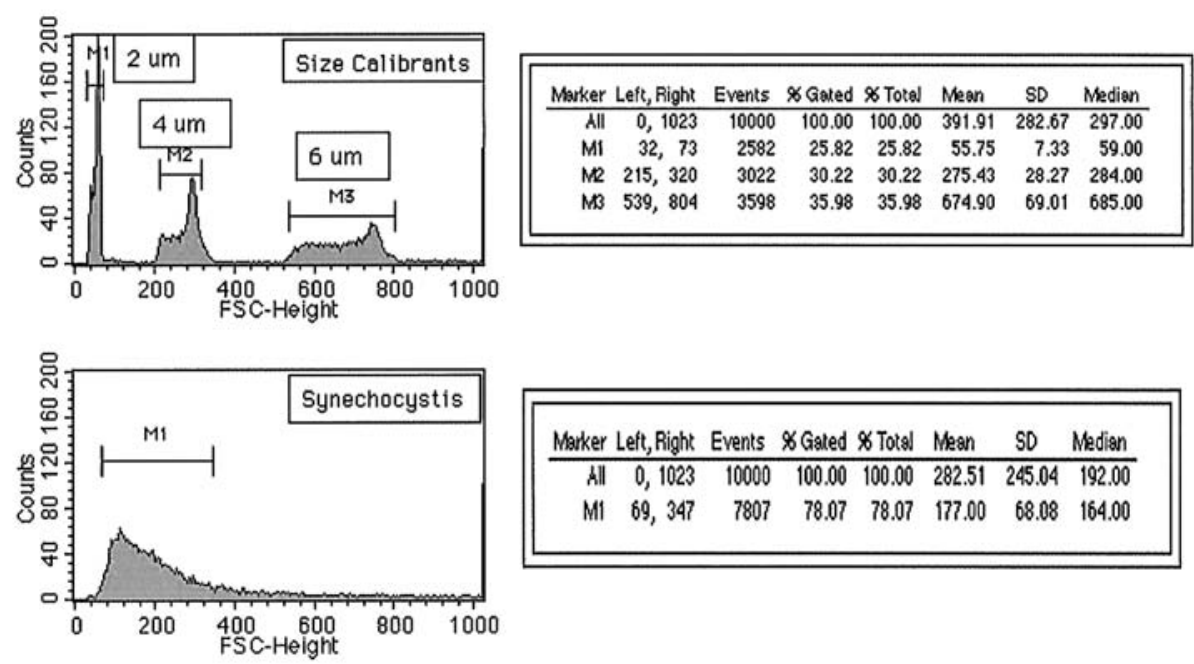

Fig. 4 Flow cytometry for Synechocystis sp. Data on the upper figure, size standard. Data on the lower figure, Synechocystis sp. 

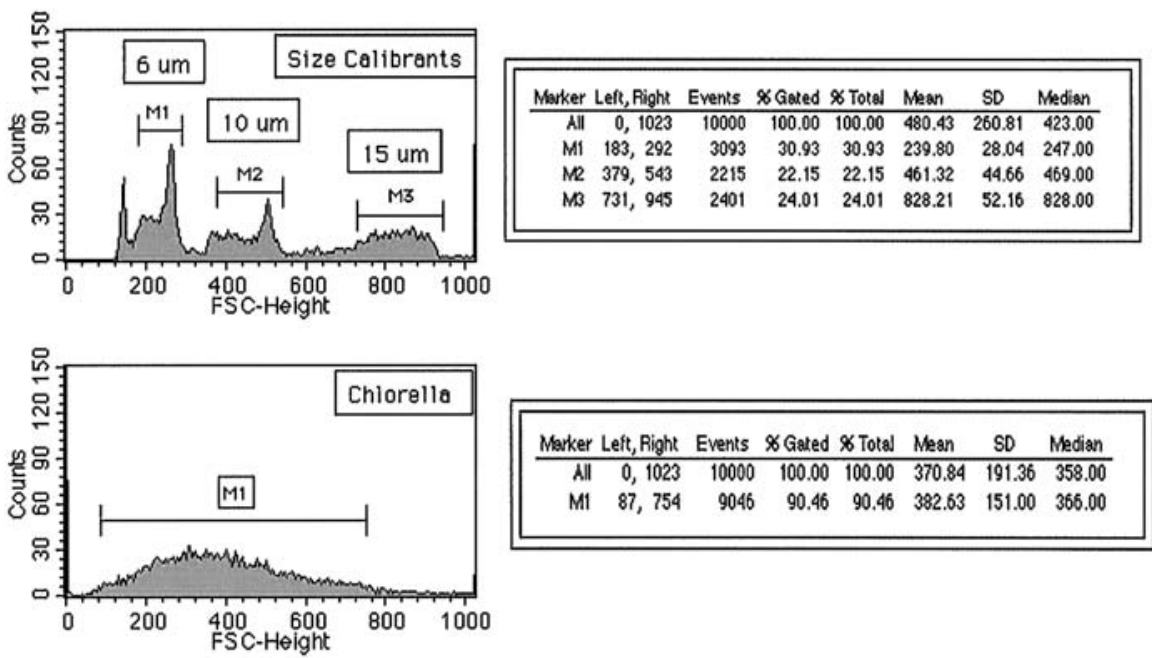

Fig. 5 Flow cytometry for Chlorella kessleri. Data on the upper figure, size standard. Data on the lower figure, Chlorella kessleri.

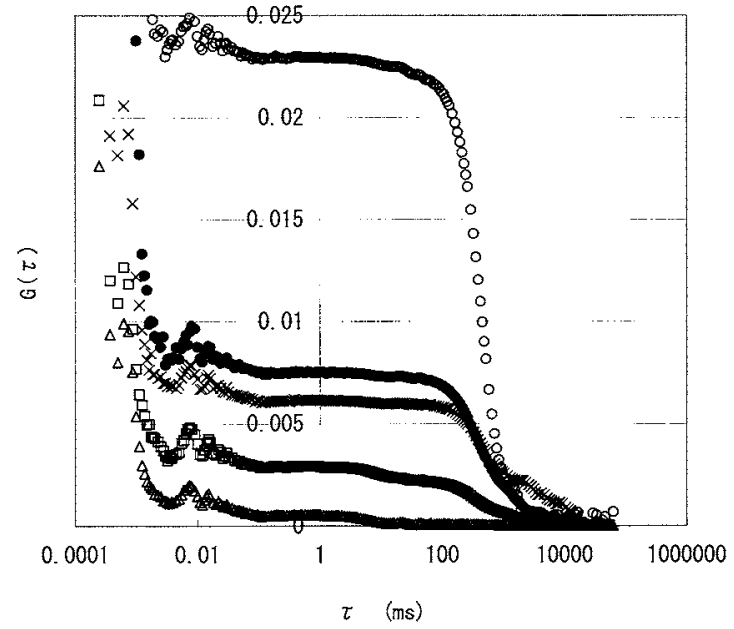

Fig. 6 FCS functions for standard beads and their mixture. 0 Standard bead $(10 \mu \mathrm{m}$ in diameter); $\bullet$, standard bead $(6 \mu \mathrm{m}$ in diameter); $\square$, standard bead ( $4.5 \mu \mathrm{m}$ in diameter); $\triangle$, standard bead $(2$ $\mu \mathrm{m}$ in diameter $) ; \times$, mixture of standard beads $(2$ and $10 \mu \mathrm{m}$ in diameter). The data are average values of five measurements.

suitable to determine the size of dyes, DNA, and proteins with an approximate volume of $10^{-6}-10^{-9} \mathrm{fL}$, and to observe the movement of substances inside planktonic cells. ${ }^{27,28}$ However, in order to observe an entire planktonic cell, the optical configuration applied in standard fluorescence spectrometry (approximately 5 orders greater than that applied in confocal analysis) can be used.

In the case of FCM, forward and side scattering, fluorescent luminescence, and in some cases, electronic pulse conductance are utilized for particle speciation; the signals generated in a stream of single particles, including plankton cells, during capillary flow under laminar flow conditions were analyzed for particle identification. Although the application of simple FCS, which provides, only cell-size information, is demonstrated, the potential applications of FCS in plankton analysis, e.g., using FLOWCAM $^{\circledR}$, can be significant.

\section{References}

1. K. Fujiwara, S. Hirokawa, and M. Aoki, Anal. Sci., 2007, 23, 1237.

2. A. Varriale, M. Rossi, M. Staiano, E. Terpetshnig, B. Barbieri, M. Rossi, and S. D'Auria, Anal. Chem., 2007, 79, 4687.

3. I. Langowski, Methods Cell Biol., 2008, 85, 341.

4. Calin C. Guet, L. Bruneaux, T. Min, D. Siegel-Gaskins, I. Figueroa, T. Emonet, and P. Cluzel, Nucleic Acids Res., 2008, 36, e73/1:AN 2008:847299.

5. H.-C. Yeh, C. M. Puleo, Y. Ho, V. Bailey, J. Vasudev, T. C. Lim, K. Liew, and T.-H. Wang, Biophys. J., 2008, 95, 729.

6. H. He, C. Xie, and J. Ren, Anal. Chem., 2008, 80, 5951.

7. J. Ries, E. P. Petrov, and P. Schwill, Biophys. J., 2008, 95, 390.

8. L. Aitziber, G. Lois, E. Sherman, C. S. O'Hern, L. Regan, and G. Haran, J. Mol. Biol., 2008, 382, 203.

9. R. Lasserre, X.-J. Guo, F. Conchonaud, Y. Hamon, O. Hawchar, A.-M. Bernard, S. M. Soudja, P. F. Lenne, H. Ligneault, D. Olive, G. Bithmuth, J. A. Nunes, B. Payrastre, D. Marguet, and H.-T. He, Nat. Chem. Biol., 2008, 4, 538.

10. T. Kogure, S. Karasawa, T. Araki, K. Saito, M. Kinjo, and A. Miyawaki, Nat. Biotechnol., 2006, 24, 577.

11. T. Torres and M. Levitus, J. Phys. Chem. B, 2007, 111, 7392.

12. R. H. Berg and R. N. Beachy, Methods Cell Biol., 2008, 85, 153.

13. A. Garcia-Marcos, S. A. Sanchez, P. Parada, J. S. Eid, D. M. Jameson, M. Ramacha, E. Gratton, B. Enrico, and J. P. G. Ballesta, Biophys. J., 2008, 94, 2884.

14. Z. Petrásek and P. Schwille, Chem. Phys. Chem., 2008, 9, 147.

15. I. Gombos, G. Steinback, I. Pomozi, A. Balogh, G. Vámosi, A. Gansen, G. László, G. Garab, and J. Matkó, Cytometry A, 2008, 73A, 220.

16. Z. Gryczynski, Biophys. J., BioFAST, published on Jan. 4, 2008, PMID 18178654.

17. B. J. Trask, G. J. van den Engh, and J. H. Elgershwizen, Cytometry, 1982, 2, 258. 
18. J. C. Peeters, G. B. Dubelaar, J. Ringelberg, and J. W. Visser, Cytometry, 1989, 10, 522.

19. G. B. Dubelaar, A. C. Groenewegen, W. Stokdijk, G. J. van den Engh, and J. W. Visser, Cytometry, 1989, 10, 529.

20. J. F. Keij, A. C. Groenewegen, G. B. Dubelaar, and J. W. Visser, Cytometry, 1995, 19, 209.

21. M. E. Sieracki, T. L. Cucci, and J. Nicinski, Appl. Environ. Microbiol., 1999, 65, 2409.

22. Y. Zhang, J. T. Bahns, Q. Jin, R. Divan, and L. Chen, Anal. Biochem., 2006, 356, 161.

23. M. E. Sieracki, C. J. Gobler, T. L. Cucci, E. C. Their, I. C. Gilg, and M. D. Keller, Harmful Algae, 2004, 3, 459.
24. C. J. Gobler, N. J. Buck, M. E. Sieracki, and S. A. SañudeWilhelmy, Estuarine Coastal Shelf Sci., 2006, 68, 127.

25. M. Gösch, H. Blom, J. Holm, T. Heino, and R. Rigler, Anal. Chem., 2000, 76, 3260.

26. B. H. Kunst, A. Schots, and A. J. G. Visser, Anal. Chem., 2002, 74, 5350 .

27. E. Gulot, E. P. Georges, A. Brun, and M. P. Fontain-Aupart, Photochem. Photobiol., 2002, 78, 570.

28. P. Lacroix-Gueu, R. Briadet, S. Leveque-Fort, M. N. Bellon-Fontain, and M. P. Fontain-Aupart, C. R. Biol., 2005, 328, 1065. 